\title{
Context-aware and pro-active queue management systems in intelligent environments
}

\author{
Radosław Klimek \\ AGH University of Science and Technology \\ Al. Mickiewicza 30, 30-059 Krakow, Poland \\ Email: rklimek@agh.edu.pl
}

\begin{abstract}
The Ambient intelligence (AmI) paradigm refers to electronic environments which are sensitive and responsive to the presence of people. Queue systems are practically used in various institutions and commercial enterprises constituting a challenge for the intelligent environments in smart cities. The management of the customer flows guarantees elimination or reduction of the queues as well as the economic benefits which follow the clients' satisfaction of the better service quality. There has been proposed the intelligent queue management system designed as the pro-active and context-aware system basing on multiple lowlevel sensors and devices constituting the IoT (Internet of Things) network. The designed context-driven system is characterized by user friendliness, as well as the client behavior understanding to generate actions that support clients. There has been proposed the conceptual version of the system. The selected aspects of the prototype version has been simulated. This prototype can be used as the necessary experience for building the target system meeting the precise needs and assumptions typical for context-aware and pro-active system basing on IoT networks.
\end{abstract}

Index Terms-intelligent environment; context-awareness; queue; queue management system; IoT.

\section{INTRODUCTION}

$\mathbf{S}$ MART cities, which become more and more common and inevitable, are understood as the places where the modern ICT technologies are used in order to improve the quality of life for citizens. They enable the sustainable usage of resources available. There is no one scenario which helps to reach this goal. Developing context-driven applications is always hard and complex. We can see queues in many places of smart cities, enterprises, as well as many different types of clients who behave differently in numerous situations and have distinctive needs or preferences. The main aim of the intelligent queue management system is to adjust to those needs which enable to minimize the length of queues, shorten the time of customer service and increase the level of satisfaction of services. Indirectly, it can also influence the increase of sales volume.

The aim of this work is to propose the intelligent queue management system for a large store. Although, the idea of the queue management system is not a new one, many of the already existing systems, in spite of being described as "intelligent" which is a slight exaggeration, use mainly the simplest methods of people flow management. These are putting the physical barriers or informing users about the overall situation and statistics related to the already existing queues, by displaying messages on the screen. Such statistics present only the simple information: how many people visited a shop at a particular hour and how much time they spent waiting in queues. There does not exist the attempt to identify the user in existing systems, considering the present behavior or historical data, assigning him/her to the particular group to be serviced in a special way. Moreover, the traditional systems are not transparent for the user, that is clients are usually in a certain interaction with the system.

The dynamic nature of a typical context-aware system is shown in Figure 1, see also [1], where different phases are repeated periodically sensing activities and to generating proper reactions enabling to operate in a smart environment. The physical world and the context-awareness software con-

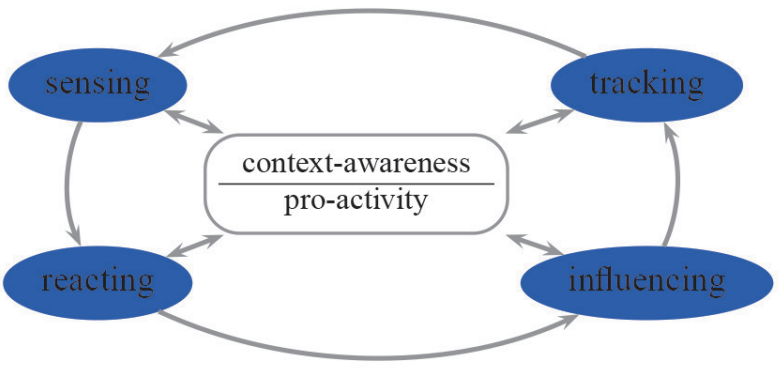

Fig. 1. Context-aware and pro-activity systems

stitute the smart environment. It contains different sensors, or other IoT (Internet of Things) equipment, distributed in the whole physical area. Smart applications enable understanding context [2], and provide pro-activity, that is act in advance to deal with future situations or actions, see [3], [4], [5], [6], [7].

There is a need, that also follows the development of the ICT technologies, of creating a really intelligent system which, in a non-intrusive way, would understand people behavior, categorize them, and manage the queues in a pro-active way. It would recognize the needs and expectations as well as supports the people who found themselves in this environment. It needs to be emphasized, that the queue management system can be implemented not only for needs of shopping mall but also for the objects such as enterprises, airports, different offices and many other places.

The system, proposed in this work, has a conceptual character, however, the analysis of the available technological solutions from the ICT range proves its technical feasibility. 
There has been developed the prototype and the simulation studies have been carried out. The prototype of the system can be furtherly developed and improved by implementing the new details for example related to reasoning, ontological reasoning, especially helpful in case of different methods of the clients identification, as well as developing different identifying components (biometric data, mobile phone identifier etc.), designing the software architecture and making all other improvements. The prototype can be used as the reference point for creating the system which would be correctly located within a particular instance and needs.

There are many works considering and discussing queue management systems. Moreover, there are also commercial systems for the line management. However, there is lack of works for smart systems that works in intelligent environments, that is systems that understand human behavior providing context-aware and pro-active actions supporting customers and inhabitants. Such systems are mandatory ingredient for smart cities. Work [8] discusses queues in the context of a smart parking system. The aim of the proposed algorithm is to control traffic. Work [9] provides methods for queuing delays when using RFID systems but they don't recognize human behavior. Work [10] also don't discuss behavior aspects while new message systems are introduced. In work [11], a ticket dispenser is used. This paper follows works [12], [1] which concern observing behaviors of users/inhabitants and modelling logical specifications understood as user preferences.

\section{BASIC ASSUMPTIONS AND REQUIREMENTS}

By a queue we understand an impermanent community of people which is created when waiting for the particular event such as serving. The formation of a queue is usually related to the small number of resources/people offering the service of serving. The members of the queue are usually handled in the set order, most often it is the FIFO rule, and later they leave the queue.

It is presumed that there exist the entrance devices which enable to identify people and events. Among them are:

- camera: equipped with the necessary software which enables to record and identify the biometric data of clients who enter the object and stay inside it, the new and old clients which means having the history of their presence in a particular object;

- GPS sensors: installed in the shopping trolleys and helping to monitor their location together with the client;

- scanners of the bar code embedded into the client's trolley;

- cameras, together with the software, built-in the object and helping to observe the characteristic way of moving of a client (elderly person, disabled person, person with children);

- thermal cameras detecting the increased body temperature (which suggests for example stress, haste but also the illness);

- cameras located near the cash registers which estimate the number of people waiting in a queue;
- sensors detecting the Bluetooth and GSM devices and estimating the number of people waiting in a queue as well as performing the supporting tasks. Such devices are the part of most of the private mobile phones.

The information and messages from system are introduced to different output devices such as:

- mobile applications which suggest the client a particular behavior, giving the number of the cash desks where he/she should go;

- displayer on the shopping trolley informing about the number of free cash registers;

- "help" button on trolley calling the shop assistant and helping in case of problems with the finalization of transaction (elderly people);

- publicly available displayer at the beginning of each cash register, as well as all other displayers in this part of store.

The type of data used for analysis can be: age, pregnancy, body posture, weight, clothing, height, body temperature, blood pressure, heart rhythm, amount of shopping. All those types of data are stored in a way which is transparent for a client.

The system monitoring the queue management system has a lot of information about the current situation in a monitored object, performs the basic measurements, analyzes the historical data which help to describe the preferences of regular clients. The basic parameters of the current situation in monitored object are:

- the number of people entering the store;

- the number of people who stay inside;

- the number of people classified into a certain category for example: elderly people, disabled people, mothers and families with small children, pregnant women, customers who usually do big shopping (those who have a substantial financial input), sick people and those whose body temperature is higher than the average one;

- the length of the particular queues;

- the average time of waiting in the particular queues, divided into the queues of the special meaning;

- the total time of waiting etc.

In order to estimate the length of a queue there are used different sources and methods from the video cameras to detecting the Bluetooth devices. However, the last method has its own limits related to the fact that in many mobile phones this function is switched off. On the other hand, it can be useful in describing the time of waiting in every queue, when we assume that at least some people have this function in an active mode, and observing the movement of the queue. Similar remarks concern the GMS system emitting the signal to the nearest BTS station (Base Transiver Station).

The aim of the whole system, apart from the typical tracing of the clients' activity and their distance from the cash registers, is also detecting the distinctive types of behaviors which enable to redirect some people to the cash registers specially designated for their needs. It can be performed on the basis of the historical behaviors analysis, if they are 


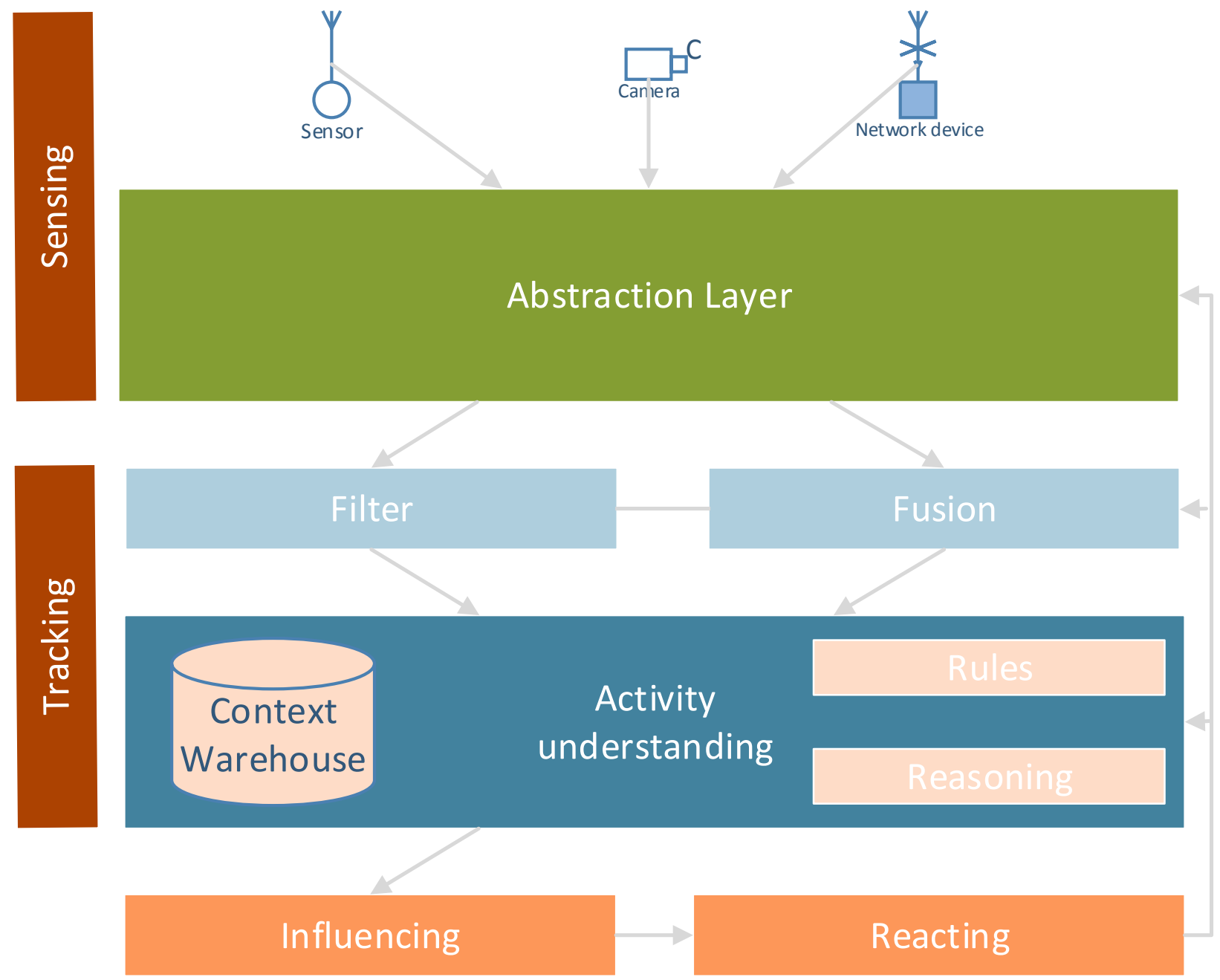

Fig. 2. Basic system architecture for activity understanding and context reasoning

available for the particular clients. This type of system, which is sensitive and context-aware and is characterized by the proactive functioning, aims to increase the level of satisfaction among clients, adjust the selling offer and consequentially increase the trading volume.

The direct objective of the system is to

- shorten the queues, or to reduce queue time, balancing the distribution of customers;

- allocate customers to specialized queues/desks in which they will be better served;

- increase customer satisfaction.

After identification and recognition of the client there are analyzed the examples presented below:

1) the person is a regular client (possibility to offer discounts and better standard of service, shorter queues, personal assistants);

2) the size of the previous shopping (discounts, redirecting to the cash register for special customers, personal assistants, possibility of shopping delivery);
3) the most frequently chosen categories (household chemistry, groceries, alcohol and others - information about sales, discounts), buying the luxury products (better quality of service);

4) elderly people, pregnant women, people with children, disabled people (special cash desks);

5) when client moves quickly or walks slowly, seemingly without a special purpose (people in hurry are redirected to the shorter queues or the special queues).

There are a few types of cash registers:

1) special cash desk adjusted to the needs of disabled or elderly people (bigger and wider driveway, bigger displayers);

2) cash desks for people with small children;

3) cash desks for people with luxury products who are the regular customers;

4) cash desks for fast service of people with a few products;

5) special cash desks for a particular type of products (for example electronics); 


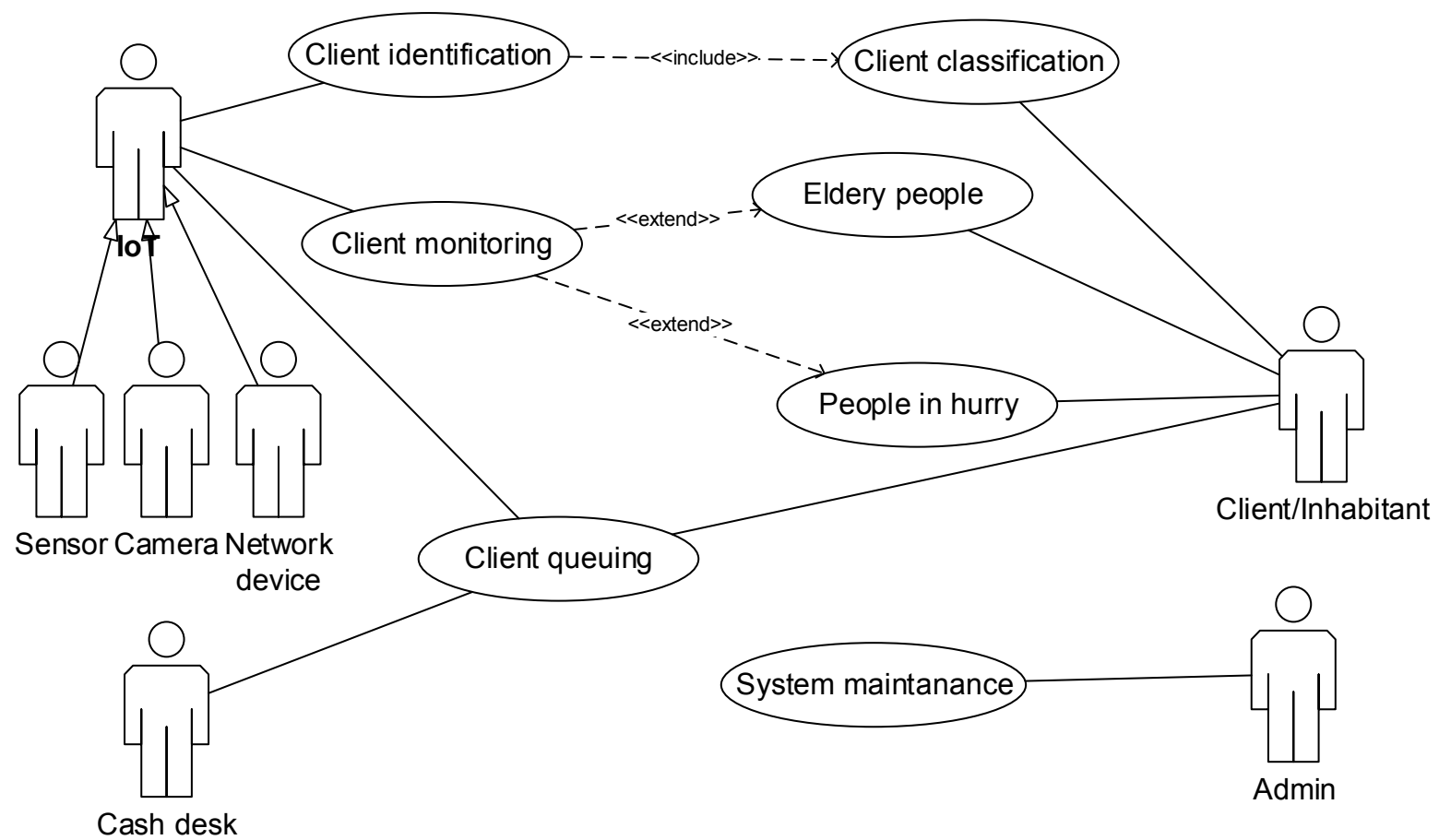

Fig. 3. Intelligent queue management system

6) normal cash registers.

\section{ARCHITECTURE}

The idea of context is well established and understood in pervasive computing. Context is "...any information that can be used to characterize the situation of an entity. An entity is a person, place, or object that is considered relevant to the interaction between a user and an application, including the user and applications themselves" [13]. Thus, contextawareness is an ability of sensing and reacting on the environment. Sensing and context understanding are necessary and of critical importance for pro-active decisions.

The overall architecture for context-aware system that understood inhabitants activities, performing context reasoning processes is shown in Fig. 2, as an adaptation of similar ones in works [14], [1]. The Context Warehouse captures information engineering, that is it allows:

- to show all the clients attached to the monitored area,

- to provide a consolidated picture of data,

- to capture and provide access to meta data,

- to provide capability for data sharing,

- to merge historical data with current data,

- a deeper understanding of what each customers is,

- how to reconcile different views of the same objects,

- to see if a customer begins behaving uncharacteristically,

- improve quality of data, etc.

Signals are obtained from the environments. Industrial cameras also produce environmental information. Network devices, that is signals emitted by Bluetooth, GSM, and Wi-Fi networks, allows to identify clients. Abstraction layer hides some implementation and hardware details. In other words, it translates information between different levels. Filter allows block or remove some information. Fusion enables merging of separate elements into a unified whole. Activity recognition and understanding allows to provide smart decision, that is to influence the inhabitants, or clients, in the environments. Context warehouse is a repository for all information, both historical and present, concerning objects in the monitored environments. Rules are how to process and conduct some recognized information. Reasoning enables drawing of inferences or conclusions automatically. Sensing means gathering and abstracting raw information concerning monitored environments. Tracking is a process of mining information on observed activities. Influencing provides smart decision. Reacting implements these decisions in the monitored environment.

\section{USE CASES AND SYSTEM SCENARIOS}

The basic use diagram for the queue management system is presented in Fig. 3. The case "Client identification" is responsible for identification of every new client. The identification is based on the available biometric data but also, in an alternative case, all other available data emitted by Bluetooth, GSM, or Wi-Fi network. The use case "Client classification" is obligatorily included in "Client identification" and results in precise analysis of the history of the system and on the basis of the historical data enables the current classification of the client. The use case "Client queuing" provides the final customer service at the cash register such as counting the final amount, updating the information about client in the 

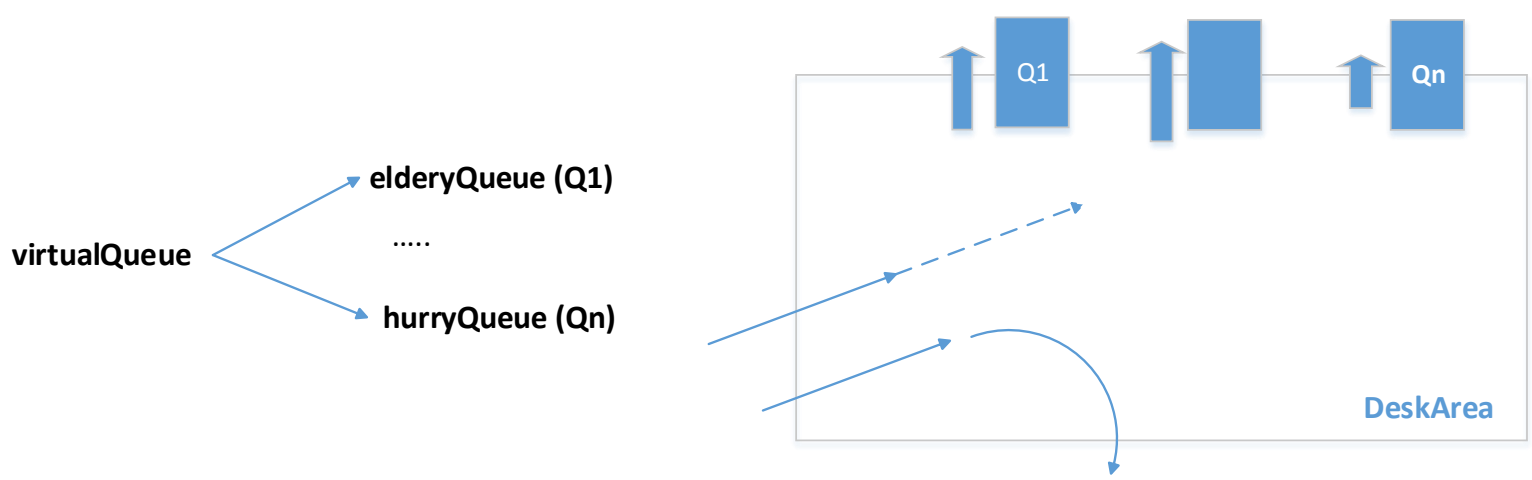

Fig. 4. Queue management system: the queue structure (left) and the desk area (right)

TABLE I

USE CASE FOR ELDERLY PEOPLE

UC name: "Eldery people"

Precondition: identification of the elderly person

Scenario:

The basic course of events:

1) Identification of the new client;

2) Recognizing the client as an elderly person;

3) Sending the message to the shop workers;

4) System monitors the client's behavior;

5) Cameras inform about his/her moving in direction of cash registers;

6) System checks the length of queues;

7) System, on the basis of the predicted waiting time and the type of client, chooses the cash register;

8) Trolley displayer informs the client about the cash register number and additionally the voice message is displayed;

9) Client moves in direction of a particular dedicated cash register.

The alternative course of events:

1) Client presses "Help" button;

2) Shop assistant comes to him/her.

Postcondition: the elderly person is serviced at the cash register and leaves the monitored area.

TABLE II

USE CASE FOR PEOPLE IN HURRY

UC name: "People in hurry"

Precondition: identification of the person in hurry

\section{Scenario:}

1) Identification of the new client

2) System monitors the client's behavior and informs about his/her quick pace of movement and the higher body temperature registered by the camera;

3) Cameras inside the store inform about his/her moving in direction of cash registers;

4) System checks the length of the queues and client is directed to a particular dedicated cash register.

Postcondition: the person in hurry is serviced at the cash register and leaves the monitored area.

system, changing the client's category and all other pieces of information which build the historic data and which, in the future, will play a crucial role during the next visit and identification of the client in the object. The use case "System maintenance" enables the current system management, typical administrative tasks, switching on and off the new devices and deleting the outdated data, keeping them in an order etc.

Among the actors of the system are:

- Cash desk - subject (a person or a device) providing the customer service for the clients waiting in a queue. His/her work should enable the smooth flow of people in a queue and its fast reduction;

- Client - the single being who stays inside the monitored object and is a part of the queue or in a short defined time is going to join it;

- Administrator - the physical person who oversees and controls working of the queue management system, configures its parameters, sensors and answers the suggestions proposed by the system itself;

- Sensor - an example of a physical element (IoT) in the system which task is to record, recognize and register the signals from the environment. It provides the information which are sent to the system in order to perform the correct interpretation.

The use case "Client monitoring" is an important and basic part of system which ensures the current observation of the identified client and recognition of the moment when he/she moves in the direction of cash registers as well as directing him/her to the appropriate cash desk as the place of trade finalization. Below, there are presented the possible scenarios for the use cases, recognizing and dealing with the special cases, divided into categories. Among them are: "Elderly people" or "People in hurry". Those cases are prompted as optional from the main case. Elderly people, who have limited movement capacities, move in a different and slower way which can easily be detected by the system, see Table I.

The next example of scenario is related to people whose behavior is atypical (for example they are in hurry). Moreover, there is a possibility of using the thermal cameras, registering the changing parameters of the client such as higher temperature, haste, see Table II.

It is possible to prepare another scenarios, for example for people with children, people with small shopping, VIP clients etc. 


\section{SYSTEM PRESENTATION}

There will be presented the general system scheme. Fig 4 shows organization of the queue system. Within the monitored space there is located the cash desk area DeskArea where are the physical cash registers (both serviced and those which require the self-service). It is supposed that every client who enters this area wants to finish his/her shopping and plans to move closer to the physical cash registers. This fact is immediately detected by the system and such client (after identification) is put to the virtual queue VirtualQueue. It is not the physical queue but a certain data structure in the system where are stored all clients cash registers the time when they join any physical queue or leave the area. One of the processes is constantly checking VirtualQueue and after the classification and assumption process, also using the historical data; chooses the target queue for a client, for example: the queue for elderly people or people in hurry. The client, after choosing the queue for him/her, is monitored by the system (mobile phone, trolley displayer or the widely available displayers within the range of the system) which informs him/her about the target queue. If the client joins the physical queue, he/she is removed from VirtualQueue. If suddenly the client leaves DeskArea, for example resigns from finalization of transaction, he/she is also removed from the system. The general algorithms for the queuing system for the use case "Client Queuing" is presented in Fig. 5.

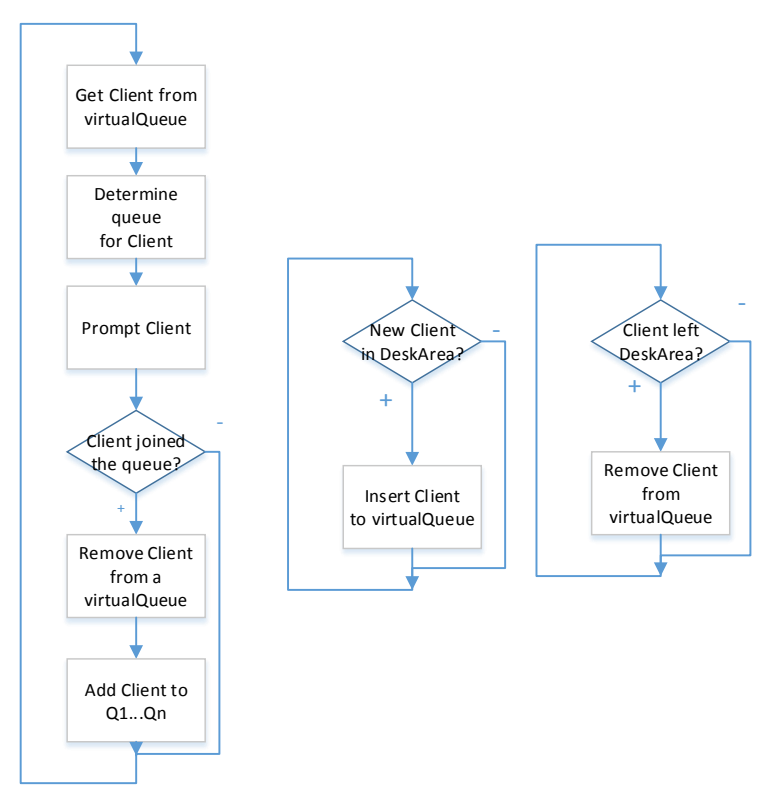

Fig. 5. Queuing, means assigning the queue to the client (left), new clients and those who leave (middle, right).

There has been created the simulation environment, or more precisely, the prototype of an application, which simulates work of the intelligent queue management system. However, this is only the beginning of work to obtain the complete system. Even if the prototype has been simplified, it still precisely portraits of how the system works. Fig. 6 presents the

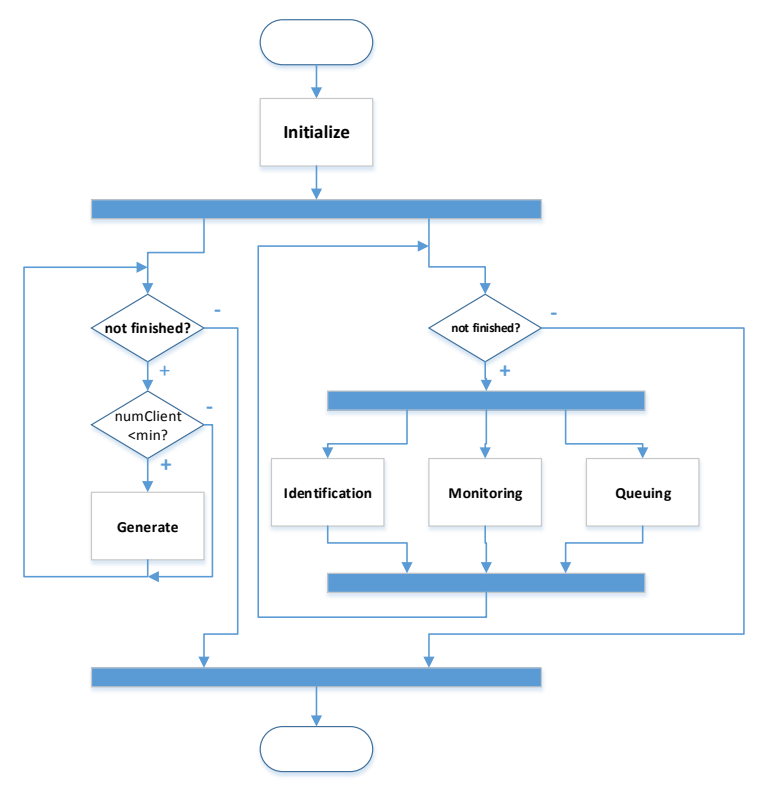

Fig. 6. Environment simulating the queue management system

general working schemata of such environment. The system is initiated, there are measured its basic parameters such as: influencing the generator of clients, random distribution, probability density and others. There is also initiated the "finished" variable which controls the end of simulation process and is set by the system administrator. The whole process of generating new clients entering the object is initiated when a current number of clients falls below a certain minimal value. The identification, monitoring and queuing are performed simultaneously.

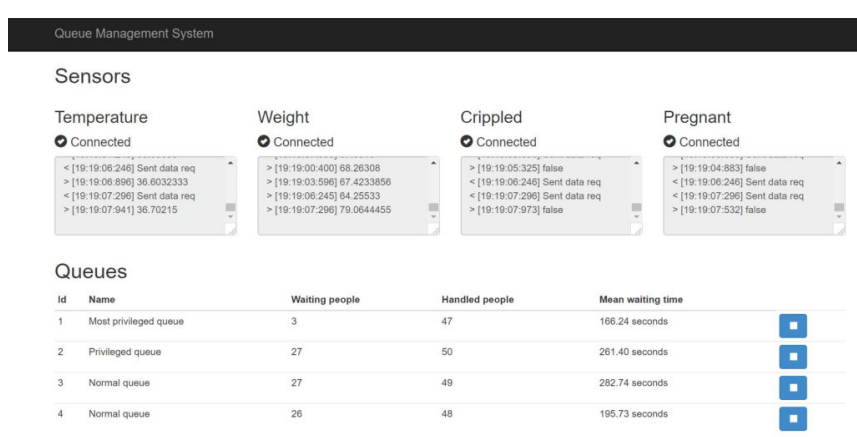

Fig. 7. Screen shot: Preview of the message exchange between sensors (IoT) and the main server.

In the simulation process, the clients are generated in a pseudo-random way, with parameters (elderly, young, physically disabled, children, men, women, pregnant women, people in hurry, regular clients, people without shopping history etc.) which enable the best possible and precise mapping of the real system. It is possible to describe the density of generating in order to map the time periods characterized by different turnout of clients (morning hours, afternoon hours).

There has been adopted the following time periods 
TABLE III

SiMULATION RESULTS

\begin{tabular}{|c|c|c|c|c|c|}
\hline \multirow{3}{*}{ Parameter } & \multirow{2}{*}{ Queue number } & \multicolumn{4}{|c|}{ Simulation time } \\
\cline { 2 - 6 } & & $30 \mathrm{~s}$ & $60 \mathrm{~s}$ & $120 \mathrm{~s}$ & $240 \mathrm{~s}$ \\
\hline \hline \multirow{3}{*}{ Number of waiting clients } & 1 & 2 & 3 & 2 & 2 \\
\cline { 2 - 6 } & 2 & 2 & 4 & 8 & 14 \\
\cline { 2 - 6 } & 3 & 2 & 4 & 7 & 14 \\
\cline { 2 - 6 } & 4 & 2 & 3 & 7 & 13 \\
\hline \multirow{3}{*}{ Number of clients serviced } & 1 & 1 & 3 & 6 & 12 \\
\cline { 2 - 6 } & 2 & 1 & 2 & 6 & 12 \\
\cline { 2 - 6 } & 3 & 1 & 3 & 5 & 12 \\
\cline { 2 - 6 } & 4 & 1 & 2 & 5 & 11 \\
\hline \multirow{3}{*}{ Average waiting time } & 1 & 15.4 & 22 & 33.3 & 42 \\
\cline { 2 - 6 } & 2 & 16.2 & 22.9 & 37.5 & 66.3 \\
\cline { 2 - 6 } & 3 & 17.3 & 26.4 & 35.5 & 68.5 \\
\cline { 2 - 6 } & 4 & 22.42 & 29.2 & 35 & 66.3 \\
\hline
\end{tabular}

and time intervals between the visits: for 06.0009.00 is RandomInRange $(10,60)$; for 09.00 14.00 is RandomInRange $(30,180)$; for 14.00 18.00 is RandomInRange $(50,800)$; for 18.00 21.00 is RandomInRange $(30,600)$; for $21.00-06.00$ is RandomInRange $(10,100)$, where the function RandomInRange $(a, b)$ means the random number $x$ in a range $x>a$ and $x<=b$. The prototype simplifies the client model to the features such as age, weight, body temperature, disability or pregnancy. When generating the people, there has been used the following statistical data: age - for $40 \%$ of population Gaussian $(25,3)$, for $60 \%$ of population Gaussian(50,10); weight Gaussian(70,10); temperature - Gaussian(36.6,0.3); and $0.16 \%$ of population for disability, $0.09770294 \%$ for pregnancy, where Gaussian function (mean, stdDev) gives back the number $x$ drawn according to the normal Gaussian distribution which has the mean value mean and standard deviation stdDev. Additionally, there has been implemented

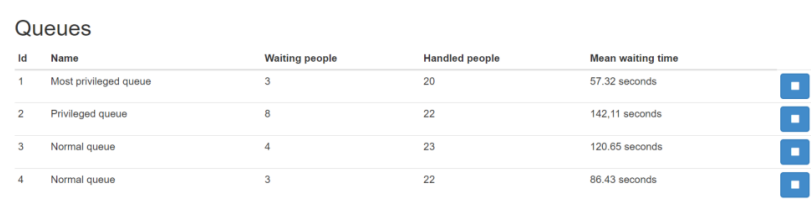

Fig. 8. Screen shot: Preview of the queue situation: name, number of people waiting, number of people serviced by a particular queue, the average waiting time and the average waiting time of an one supplicant in a queue. In the last column there is a button which stops and starts the queue.

non-deterministic time of customer service which is equal to Gaussian(90000,15000). Another implemented value is a sensor error equal to $5 \%$. Summing up the briefly presented simulation environment, it seems that the obtained environment is an appropriate framework for future works implementing the context-aware queue management system.
The exemplary screen shots presenting the system are shown in Figs. 7 and 8. Table III shows the sample results of prepared stimulation.

The further works over the system development should be carried with a special diligence according to the following non-functional requirements:

- Expansion: system needs to be designed in a way which enables its development and adding modules dealing with different spheres of controlling numerous institutions.

- Scalability: system should work smoothly in case of a sudden increase in the number of clients. The proper functioning in the period of a high burden is expected.

- Manageability: system should deploy the interfaces which help to control the queue management in a fast and effective way.

- Configurability: the system should allow the wide spectrum of configuration options - one of them is possibility of registration of the new sensors and managing the already existing ones.

- Safety: the system should be provisioned against the external attacks - it needs to use only safe and encrypted protocols.

- Stability: system should work in a stable way regardless of the environmental conditions.

The further works over the system development should also concern requirements engineering aspects [15], [16], as well as temporal issues of the system [17], [18].

\section{CONCLUSIONS}

There has been designed the queue management system in an intelligent environment. It works according to the paradigm of pervasive and ubiquitous computing. Its main task is to recognize clients who appear in the object, understand their behavior, take into account the historical data related to objects and propose the particular actions. They can cover both client support as well as simplification of the transaction finalization 
and the choice of the best store cash register. There are many different cash registers and also the customers have distinctive preferences but the main goal is the client's satisfaction and his/her safety.

The newly created simulation environment enables the initial testing of the basic principles. Both the environment as well as the system itself can be furtherly developed reaching the goal which is creation of the target system. There exist many places for the system installation and one of them could be the dean's office where the system recognizes a student, analyzes his/her current situation both in case of the whole educational process and the scholarship system, and can suggest the proper solutions and an appropriate desk.

\section{ACKNOWLEDGMENT}

I would like to thank my students Piotr Gryt, Anna Sotwin, Jakub Pelc, and Tomasz Korecki for their help and appreciate their technical skills.

\section{REFERENCES}

[1] R. Klimek, "Behaviour recognition and analysis in smart environments for context-aware applications," in Proceedings of the IEEE International Conference on Systems, Man, and Cybernetics (SMC 2015), October 9-12, 2015, City University of Hong Kong, Hong Kong. IEEE Computer Society, 2015, pp. 1949-1955. [Online]. Available: http://dx.doi.org/10.1109/SMC.2015.340

[2] A. Zimmermann, A. Lorenz, and R. Oppermann, "An operational definition of context," in Proceedings of the 6th International and Interdisciplinary Conference on Modeling and Using Context, CONTEXT'07, Roskilde, Denmark, ser. Lecture Notes in Artificial Inteligence, vol. 4635. Berlin, Heidelberg: Springer-Verlag, 2007, pp. 558-571. [Online]. Available: http://dx.doi.org/10.1007/978-3-54074255-5_42

[3] R. Klimek and L. Kotulski, "Towards a better understanding and behavior recognition of inhabitants in smart cities. a public transport case," in Proceedings of 14th International Conference on Arificial Inteligence and Soft Computing (ICAISC 2015), 14-18 June, 2015, Zakopane, Poland, ser. Lecture Notes in Artificial Intelligence, L. Rutkowski and et al, Eds., vol. 9120. Springer Verlag, 2015, pp. 237-246. [Online]. Available: http://dx.doi.org/10.1007/978-3-31919369-4_22

[4] R. Klimek and G. Rogus, "Proposal of a context-aware smart home ecosystem," in Proceedings of 14th International Conference on Arificial Inteligence and Soft Computing (ICAISC 2015), 14-18 June, 2015, Zakopane, Poland, ser. Lecture Notes in Artificial Intelligence, L. Rutkowski and et al, Eds., vol. 9120. Springer Verlag, 2015, pp. 412-423. [Online]. Available: http://dx.doi.org/10.1007/978-3-31919369-4 37

[5] R. Klimek, "Mapping population and mobile pervasive datasets into individual behaviours for urban ecosystems," in Proceedings of 15th International Conference on Arificial Inteligence and Soft Computing (ICAISC 2016), 12-16 June, 2016, Zakopane, Poland, ser. Lecture Notes in Artificial Intelligence, L. Rutkowski and et al, Eds., vol. 9692. Springer Verlag, 2016, pp. 683-694. [Online]. Available: http://dx.doi.org/10.1007/978-3-319-39378-0_58

[6] R. Klimek, L. Kotulski, and A. Sedziwy, State of the Art on AI Applied to Ambient Intelligence, ser. Frontiers in Artificial Intelligence and Applications. IOS Press, 2017, ch. Behavioural patterns from cellular data streams and outdoor lighting as strong allies for smart urban ecosystems.
[7] R. Klimek, "Understanding human behaviours in intelligent environments. a context-aware system supporting mountain rescuers," in Proceedings of 16th International Conference on Arificial Inteligence and Soft Computing (ICAISC 2017), 11-15 June, 2017, Zakopane, Poland, ser. Lecture Notes in Artificial Intelligence, L. Rutkowski and et al, Eds., vol. 10246. Springer Verlag, 2017, pp. 267-279. [Online]. Available: http://dx.doi.org/10.1007/978-3-319-59060-8_25

[8] A. Roy, J. Siddiquee, A. Datta, P. Poddar, G. Ganguly, and A. Bhattacharjee, "Smart traffic parking management using iot," in 2016 IEEE 7th Annual Information Technology, Electronics and Mobile Communication Conference (IEMCON), 2016, pp. 1-3. [Online]. Available: http://dx.doi.org/10.1109/IEMCON.2016.7746331

[9] Y. Berdaliyev and A. P. James, "Rfid-cloud smart cart system," in 2016 International Conference on Advances in Computing, Communications and Informatics (ICACCI), 2016, pp. 2346-2352. [Online]. Available: http://dx.doi.org/10.1109/ICACCI.2016.7732405

[10] F. Stancu, D. Popa, L.-M. Groza, and F. Pop, Queuing-Based Processing Platform for Service Delivery in Big Data Environments. Springer International Publishing, 2016, pp. 497-508. [Online]. Available: http://dx.doi.org/10.1007/978-3-319-32689-4_38

[11] Y. W. Lin and Y. B. Lin, "Mobile ticket dispenser system with waiting time prediction," IEEE Transactions on Vehicular Technology, vol. 64, no. 8, pp. 3689-3696, Aug 2015. [Online]. Available: http://dx.doi.org/10.1109/TVT.2014.2356644

[12] R. Klimek and L. Kotulski, "Proposal of a multiagent-based smart environment for the IoT," in Workshop Proceedings of the 10th International Conference on Intelligent Environments, Shanghai, China 30th June-1st of July 2014, ser. Ambient Intelligence and Smart Environments, J. C. Augusto and T. Zhang, Eds., vol. 18. IOS Press, 2014, pp. 37-44. [Online]. Available: http://dx.doi.org/10.3233/978-161499-411-4-37

[13] A. K. Dey and G. D. Abowd, "Towards a better understanding of context and context-awareness," in Workshop on The What, Who Where, When, and How of Context-Awareness (CHI 2000), April 2000. [Online]. Available: http://www.cc.gatech.edu/fce/contexttoolkit/

[14] D. Preuveneers, A. Ramakrishnan, T. V. Hamme, V. Rimmer, Y. Berbers, and W. Joosen, State of the Art on AI Applied to Ambient Intelligence, ser. Frontiers in Artificial Intelligence and Applications. IOS Press, 2017, ch. A survey on applying machine learning techniques for behavioral awareness.

[15] R. Klimek, "From extraction of logical specifications to deductionbased formal verification of requirements models," in Proceedings of 11th International Conference on Software Engineering and Formal Methods (SEFM 2013), 25-27 September 2013, Madrid, Spain, ser. Lecture Notes in Computer Science, R. M. Hierons, M. G. Merayo, and M. Bravetti, Eds., vol. 8137. Springer Verlag, 2013, pp. 61-75. [Online]. Available: http://dx.doi.org/10.1007/978-3-642-40561-7 5

[16] M. Grobelny, I. Grobelna, and M. Adamski, "Hardware behavioural modelling, verification and synthesis with UML 2.x activity diagrams," in 11th IFAC Conference on Programmable Devices and Embedded Systems, PDeS 2012, Brno, Czech Republic, May 23-25, 2012, Z. Bradác and F. Zezulka, Eds. International Federation of Automatic Control, 2012, pp. 134-139. [Online]. Available: http://dx.doi.org/10.3182/20120523-3-CZ-3015.00028

[17] M. Mach-Król and K. Michalik, "Validation and verification of temporal knowledge as an important aspect of implementing a temporal knowledge base system supporting organizational creativity," in 2015 Federated Conference on Computer Science and Information Systems, FedCSIS 2015, Lódz, Poland, September 13-16, 2015, M. Ganzha, L. A. Maciaszek, and M. Paprzycki, Eds., 2015, pp. 1315-1320. [Online]. Available: http://dx.doi.org/10.15439/2015F78

[18] K. Kluza, K. Jobczyk, P. Wisniewski, and A. Ligeza, "Overview of time issues with temporal logics for business process models," in Proceedings of the 2016 Federated Conference on Computer Science and Information Systems, FedCSIS 2016, Gdańsk, Poland, September 11-14, 2016., M. Ganzha, L. A. Maciaszek, and M. Paprzycki, Eds., 2016, pp. 1115-1123. [Online]. Available: http://dx.doi.org/10.15439/2016F328 